\title{
A measurement technique to determine the calibration accuracy of an electromagnetic tracking system to radiation isocenter
}

\author{
Dale W. Litzenberg, ${ }^{\text {a) }}$ Ian Gallagher, Kathryn J. Masi, Choonik Lee, Joann I. Prisciandaro, \\ Daniel A. Hamstra, Timothy Ritter, and Kwok L. Lam \\ Department of Radiation Oncology, University of Michigan, Ann Arbor, Michigan 48109-5010
}

(Received 19 November 2012; revised 19 June 2013; accepted for publication 29 June 2013; published 22 July 2013)

Purpose: To present and characterize a measurement technique to quantify the calibration accuracy of an electromagnetic tracking system to radiation isocenter.

Methods: This technique was developed as a quality assurance method for electromagnetic tracking systems used in a multi-institutional clinical hypofractionated prostate study. In this technique, the electromagnetic tracking system is calibrated to isocenter with the manufacturers recommended technique, using laser-based alignment. A test patient is created with a transponder at isocenter whose position is measured electromagnetically. Four portal images of the transponder are taken with collimator rotations of $45^{\circ} 135^{\circ}, 225^{\circ}$, and $315^{\circ}$, at each of four gantry angles $\left(0^{\circ}, 90^{\circ}, 180^{\circ}, 270^{\circ}\right)$ using a $3 \times 6 \mathrm{~cm}^{2}$ radiation field. In each image, the center of the copper-wrapped iron core of the transponder is determined. All measurements are made relative to this transponder position to remove gantry and imager sag effects. For each of the 16 images, the 50\% collimation edges are identified and used to find a ray representing the rotational axis of each collimation edge. The 16 collimator rotation rays from four gantry angles pass through and bound the radiation isocenter volume. The center of the bounded region, relative to the transponder, is calculated and then transformed to tracking system coordinates using the transponder position, allowing the tracking system's calibration offset from radiation isocenter to be found. All image analysis and calculations are automated with inhouse software for user-independent accuracy. Three different tracking systems at two different sites were evaluated for this study.

Results: The magnitude of the calibration offset was always less than the manufacturer's stated accuracy of $0.2 \mathrm{~cm}$ using their standard clinical calibration procedure, and ranged from 0.014 to $0.175 \mathrm{~cm}$. On three systems in clinical use, the magnitude of the offset was found to be $0.053 \pm 0.036,0.121$ \pm 0.023 , and $0.093 \pm 0.013 \mathrm{~cm}$.

Conclusions: The method presented here provides an independent technique to verify the calibration of an electromagnetic tracking system to radiation isocenter. The calibration accuracy of the system was better than the $0.2 \mathrm{~cm}$ accuracy stated by the manufacturer. However, it should not be assumed to be zero, especially for stereotactic radiation therapy treatments where planning target volume margins are very small. (C) 2013 American Association of Physicists in Medicine. [http://dx.doi.org/10.1118/1.4813910]

Key words: electromagnetic tracking, Winston-Lutz, star shot, calibration, radiation isocenter

\section{INTRODUCTION}

Historically, planning target volume (PTV) margins of $1.0 \mathrm{~cm}$ or more were widely used in developing external beam radiation therapy treatment plans for prostate cancer when only initial or weekly port films were available. ${ }^{1,2}$ With the introduction of ultrasound localization, implanted gold fiducial markers and cone-beam CT for daily localization, margins of $0.4-1.0 \mathrm{~cm}$ became more common. ${ }^{3}$ The introduction of continuous electromagnetic tracking has allowed intrafraction motion to be measured and corrected in real-time during treatment. With this additional level of monitoring and corrective intervention, clinical protocols have been conducted with PTV margins reduced to as low as $0.3-0.5 \mathrm{~cm}^{4}{ }^{4,5}$ As margins are reduced to this level, and dose distributions become more conformal, it becomes increasingly important to quantify the accuracy with which the localization system is calibrated to radiation isocenter to avoid systematic marginal misses.

Previous investigations of transponder localization accuracy, relative to the detector array, found standard deviations of $0.0006-0.048 \mathrm{~cm}$ about the expected position in each direction, depending on distance from the array. ${ }^{6}$ That study, however, was not conducted under clinical conditions where the array must also be localized within the treatment room by infrared cameras and the whole system calibrated to isocenter. Other investigations demonstrated the static and dynamic localization accuracy of the system relative to a calibration phantom mounted on a 4D motion stage, whose zero was set when the phantom was aligned to the room lasers, where it was assumed that the lasers coincided with radiation isocenter. ${ }^{7,8}$

In this study, we report a technique developed to investigate the systematic and random errors in calibrating the 
Calypso System (Varian Medical Systems, Palo Alto, CA) to radiation isocenter. This technique was developed as a quality assurance tool for a multi-institutional protocol using electromagnetic guidance for hypofractionated prostate radiation therapy. In this protocol a $0.3 \mathrm{~cm}$ uniform margin is used to create the PTV. While the manufacturer specifies that the tracking system has an accuracy of $0.2 \mathrm{~cm}$ (Ref. 9) or better, a $0.2 \mathrm{~cm}$ calibration error in the system would leave little room for other positional errors during treatment on this protocol.

The proposed method extends the basic star shot and "Winston-Lutz" techniques ${ }^{10,11}$ of finding radiation isocenter to also find "radiofrequency isocenter." Rather than using a fixed radio-opaque ball placed at isocenter, a fixed radiofrequency (RF) transponder is placed near isocenter. This provides both a fixed radiographic and RF reference that serves as the intermediary for finding the offset between radiation isocenter and the calibrated origin of the RF localization system. In addition, by using a MLC shaped field rather than a stereotactic cone, modified forms of the star and spoke shot tests for gantry and collimator rotations are inherent to the technique. ${ }^{12,13}$ This allows the size of radiation isocenter to be measured simultaneously. It should be noted that many of the concepts used in this technique are not new, but have been reported previously as a means to investigate gantry sag, the size of radiation isocenter and its coincidence with mechanical isocenter. ${ }^{14-19}$ The technique also provides a method for institutions participating in this clinical study to independently check that the accuracy of the radiofrequency tracking system conforms to the $0.2 \mathrm{~cm}$ tolerance recommended for nonradiographic localization and positioning system by AAPM Task Group 147. ${ }^{20}$

\section{METHODS AND MATERIALS}

In this section, we first describe the measurements, followed by the rationale for the design, techniques, and analysis that are used to find the offset between radiation and RF isocenter.

\section{A. Measurements}

First, the treatment room lasers are adjusted to isocenter using a Mechanical Isocenter Standard (MIS) which is part of a Radionics (Integra NeuroSciences, Burlington, MA) BRW (Brown-Roberts-Wells) stereotactic radiosurgery alignment system. The electromagnetic tracking system is then calibrated to isocenter using the manufacturers recommended technique, which is based on alignment of the calibration phantoms to the room lasers. The "tennis racket" is removed from the treatment table, or the table moved away from the gantry, so that it does not appear in subsequent megavoltage portal images. A single transponder is then placed near isocenter in the treatment field. The position of the isocenter transponder is measured electromagnetically, and then the tracking array is moved so that it does not appear in the portal images.
With the transponder near isocenter 17 treatment fields are imaged as follows. First, a treatment field of dimension $3 \times 18 \mathrm{~cm}^{2}$ is imaged with the gantry at $90^{\circ}$ and the collimator at $135^{\circ}$. (This will be used in conjunction with the second image to determine the magnification at the imager plane as described below.) Then, four portal images of a $3 \times 6 \mathrm{~cm}^{2}$ field are taken at each of the cardinal gantry angles $\left(90^{\circ}, 0^{\circ}\right.$, $270^{\circ}, 180^{\circ}$ ) with collimator rotations of $135^{\circ}, 45^{\circ}, 315^{\circ}$, and $225^{\circ}$. The fields are ordered to minimize gantry motion first, then collimator motion.) All treatment fields are shaped using the MLC with the jaws placed $0.3 \mathrm{~cm}$ behind each MLC field edge. The portal imager is placed at $150 \mathrm{~cm}$ from the source to avoid collisions with the treatment table and magnify the image of the transponder.

The tracking array is then repositioned and a second electromagnetic measurement of the transponder position is made to verify that it did not move during imaging. The $17 \mathrm{im}-$ ages are then exported from the record and verify system. Additionally, the transponder position data are exported from the tracking system for analysis. It should be noted that the position data for an individual transponder used in this technique is not available from the standard clinical configuration of the electromagnetic tracking system, and was made available only through a research agreement with the manufacturer.

\section{B. Experimental design and technique}

\section{B.1. Transponder placement and function}

A test patient is created within the tracking system with one transponder at isocenter and another placed $3 \mathrm{~cm}$ inferiorly. Only the transponder placed near isocenter is needed for these measurements, but the second transponder must be present for the electromagnetic tracking system to function in its commercial clinical configuration. The position of the inferior transponder is chosen so that it will not appear in the $3 \mathrm{~cm}$ wide portal images. The third transponder commonly used for clinical prostate tracking is excluded in the software configuration for this test patient.

The two transponders are fixed $3 \mathrm{~cm}$ apart in a small plastic straw that will not appear in MV portal images. As shown in Fig. 1, the straw is cantilevered into the imaging field on a rigid nonconducting arm $\left(\frac{1}{2} \times \frac{1}{2}\right.$ in. square nylon rod depicted) such that one transponder is roughly near isocenter. The transponder is oriented so that its long axis is in the superior-inferior direction, parallel to the gantry rotation axis. In this cylindrically symmetric orientation it will have the same appearance on all images regardless of gantry angle. This simplifies the image processing algorithm used to find the center of the inductor in the transponder.

The transponder is absolutely fixed in space, and as such, it is the reference point for all image-based measurements. All beam edges in the images are found relative to the position of the fixed transponder. This choice of reference, rather than a fixed location on the imager plane or relative to the beam (such as central axis), eliminates the effects of gantry and 


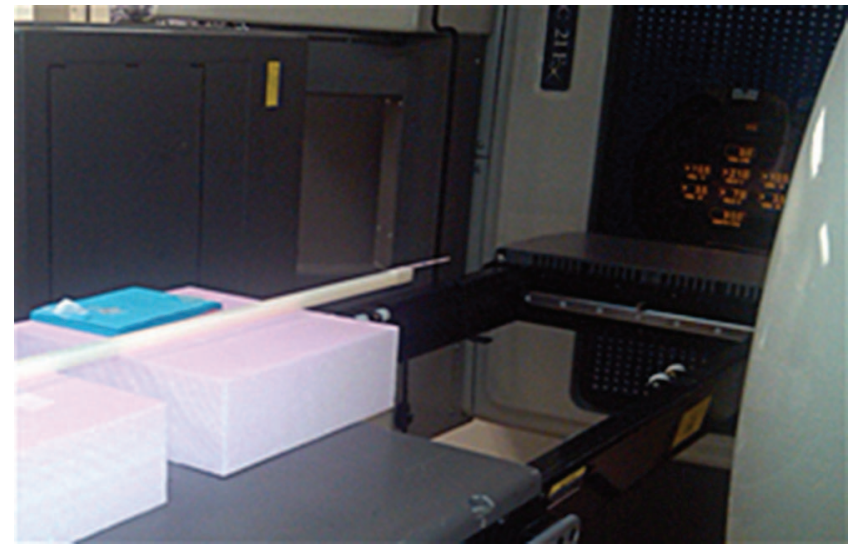

FIG. 1. Two transponders placed in a straw cantilevered into the imaging field with the table's "tennis racket" removed.

imager sag in the results. Because the MV treatment beam is used for imaging, rather than the orthogonal $\mathrm{kV}$ imaging system, radiation isocenter may be found in direct and absolute relation to the position of the transponder. This eliminates uncertainties associated with quantifying the offsets between the imaging systems (both $\mathrm{kV}$ and $\mathrm{MV}$ ) and radiation isocenter. Furthermore, it also eliminates the uncertainty in identifying a secondary reference relative to the transponders, such as the radiographic ball at the center of the Calypso monthly QA phantom. When MV imaging is used, the inductor produces a much stronger radiographic signal than the surrounding glass capsule, as shown on the right side of Fig. 2. As a result, the inductor is easily isolated with an appropriate image threshold value as described below. Because the center of the inductor and ferrite component of the transponder is the center of the re-emitted RF dipole signal detected by the tracking array, ${ }^{21} \mathrm{MV}$ imaging removes the bias caused by any offset of this inductor within the transponder's glass capsule.

\section{B.2. Image analysis}

All image and data analysis was automated and performed using IgorPro v6.21 (WaveMetrics Inc., Lake Oswego, OR). In each image, the location of each specific collimation edge as well as the center of the transponder was found, as described in Secs. 2.B.2.a and 2.B.2.b.

2.B.2.a. Collimation edges. Collimation edges are defined as the $50 \%$ intensity level between a nominal maximum beam value and the average background level for each image. The nominal maximum beam intensity is chosen as the 98th percentile value of the sorted pixel intensity values, to remove the impact of bad pixels. The average background level is chosen to be the 20th percentile value of the sorted intensity values. The X1 and X2 (formed by the rounded leaf tips) and Y1 and Y2 (formed by leaf sides) collimation edges are found starting about $0.25 \mathrm{~cm}$ away from each corner as shown in Fig. 2. Lines are then fit to each collimation edge.

2.B.2.b. Transponder location. The central region of the beam, defined as a square of $1.6 \mathrm{~cm}$ on a side (in the imager plane), is searched to find the center of the copper inductor coil of the transponder. The region is smoothed and the average of the first and last three pixel values in each row are subtracted off all values in that row to remove the impact of differential gain in the imager electronics. The standard deviation of the intensity values in the region is then found. Pixel values that differ by more than one and a half standard deviations are classified as being in the inductor coil of the transponder. The first moments of the intensity values of pixels within the transponder are used to find the center of the inductor within the transponder in each direction, as described below. The line fits to the collimation edges are then transformed to be relative to the center of the inductor coil in the inductor, making changes in imager position due to sag irrelevant.

Simulations show that when the full-width-half-maximum (FWHM) of the simulated Gaussian profile of the transponder is greater than the pixel size, the uncertainty in locating the center of the profile is less than $1 \%$ of the pixel width. Image noise then becomes the dominate source of uncertainty. Simulations additionally show that the uncertainty in identifying the center of the profile, $\sigma_{t}$, increases linearly with the noise in the image, $\sigma_{I}$, and inversely with the square root of the number of lines on which the profile appears. In the experimental setup presented here, the FWHM of the transponder profile was found to be $0.325 \mathrm{~cm}$ (as fit by a Gaussian), which is much larger than the pixel size of $0.0784 \mathrm{~cm}$. Image noise, $\sigma_{I}$, in the middle of the $3 \times 6 \mathrm{~cm}^{2}$ field, was found
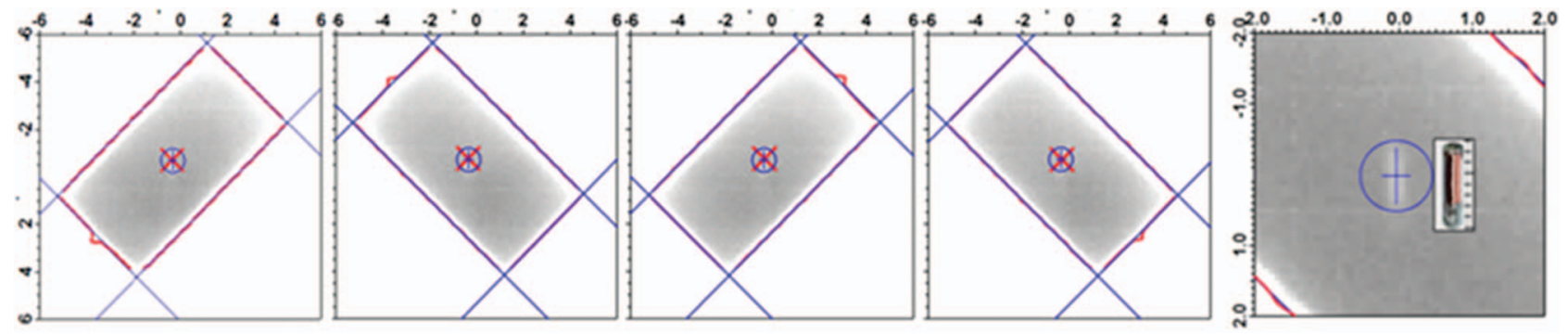

FIG. 2. At each cardinal gantry, the same $3 \times 6 \mathrm{~cm}^{2}$ field is imaged at collimator angles of $135^{\circ}, 45^{\circ}, 315^{\circ}$ and $225^{\circ}$ and shown from left to right in the images above (units on the image axes are $\mathrm{cm}$ ). The $50 \%$ field edges are found for each collimation edge, as shown by the red (bold) lines. The blue (thin) lines show line fits to each $50 \%$ field edge. The inductor's center within the transponder is shown in the right image, where a picture of a transponder is shown to scale for reference. 


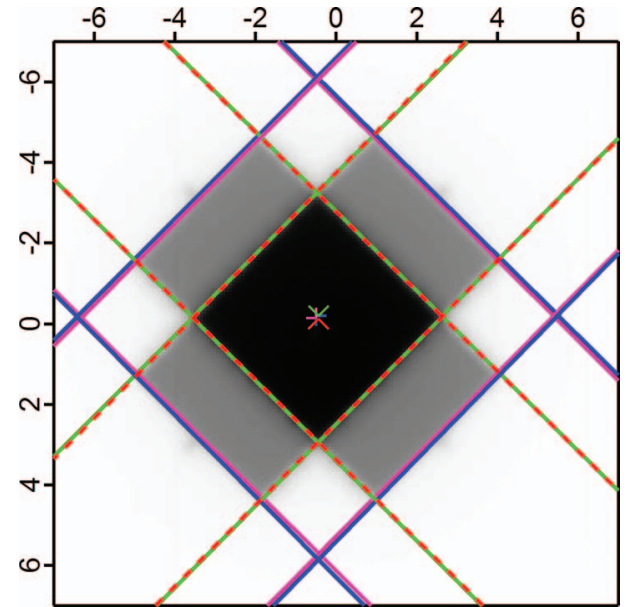

FIG. 3. A virtual square field is constructed for each collimation edge whose center defines the collimator rotation axis for that edge.

to be $0.0035 \%$. Incorporating these empirical values into the simulation yields a less than $0.02 \mathrm{~cm}$ uncertainty in identifying the center of the coil.

\section{B.3. Collimation rotation axis and radiation isocenter}

For greatest accuracy, it cannot be assumed that the X1 and $\mathrm{X} 2$ collimation edges are perfectly symmetrical about the rotation axis of the collimator. Instead, the line fits to the X1 collimation edges, for example, from each of the four collimator rotation angles at a given gantry angle are used to form a virtual square field for that collimation edge, as shown in Fig. 3. The center of the square is defined as the collimator rotation axis for that collimation edge, relative to the transponder. This is repeated for each collimation edge resulting in four collimator rotation axes for each gantry angle, as shown in Fig. 3. This is repeated at each of the four gantry angles to obtain 16 collimator rotation axis rays that pass through, or bound, the radiation isocenter uncertainty volume, as shown in Fig. 4. The center of this rectilinear bounded volume is defined here to be the central position of radiation isocenter (black sphere in Fig. 4), relative to the fixed transponder which is at the origin. The size of the radiation isocenter uncertainty volume can then be directly determined by the dimensions of the bounded volume.

\section{B.4. Spatial calibration}

To remove the image magnification from the results the first two images (both imaged at a gantry position of $90^{\circ}$ and collimator rotation of $135^{\circ}$ ) are used to determine the magnification factor. The first image is of an $(\mathrm{X}, \mathrm{Y})=3 \times 18 \mathrm{~cm}^{2}$ field, while the second field is $3 \times 6 \mathrm{~cm}^{2}$. The $X$ field size cannot be used due to the undetermined transmission through the rounded leaf tips. However, in the Y direction, the MLC leaf width may be used as a ruler whose dimensions are manufactured with high accuracy and precision when projected to the isocenter plane. Because the tongue and groove on the sides of the leaves protrude into the treatment field, a difference tech-

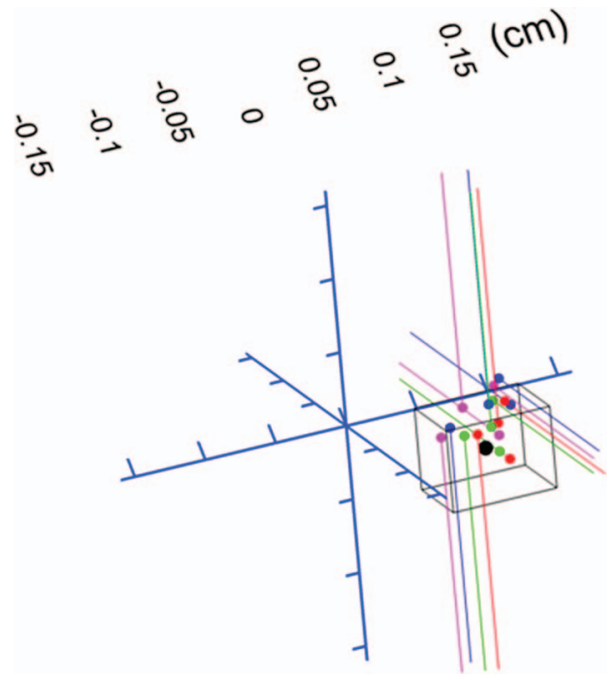

FIG. 4. Rotation axes for each collimator edge pass through or bound radiation isocenter (rectilinear box). The radiographic center of the inductor coil within the transponder defines the origin.

nique is used to remove this effect. The length of each field is found in the $\mathrm{Y}$ direction and the difference between them is found in the imager plane. This known distance at isocenter is then measured on the imager plane using the pixel dimensions which are fabricated with submicron resolution. The ratio of the difference in field length on the imager to the known size at isocenter (12 cm in this case) gives the magnification. This technique was compared to direct imaging measurements of a precision-ground machinist rule placed at isocenter and found to agree within $0.3 \%$.

\section{B.5. Offset between radiation and $\mathrm{RF}$ isocenter}

The vector position of the transponder, $\mathbf{T}_{\mathrm{C}}$, is measured electromagnetically, with respect to the calibrated RF isocenter of the tracking system. The vector position of radiation isocenter, relative to the transponder, $\mathbf{I}_{\mathrm{T}}$, is then added to the position of the transponder, to find the position of RF isocenter, $\mathbf{C}_{\mathrm{I}}$, relative to radiation isocenter. The magnitude of $\mathbf{C}_{\mathbf{I}}$, therefore, gives the offset between radiation isocenter and $\mathrm{RF}$ isocenter. This is illustrated in Fig. 5, where the origin of the coordinate system is the radiographic position of the inductor coil in the transponder, since all measurements are made relative to the fixed transponder. The dark dot, I, shows the position of radiation isocenter and the light dot, $\mathbf{C}$, illustrates $\mathrm{RF}$ isocenter.

Note that the result is independent of the position of the transponder, which serves primarily as a fixed reference relative to both radiation and RF isocenter. This independence was verified to within measurement uncertainty in consecutive measurements with the transponder moved between imaging datasets. The magnitude of the calibration offset, $\mathbf{C}_{\mathbf{I}}$ in Fig. 5, remained constant to within $0.011 \mathrm{~cm}$ when the transponder position, $\mathbf{T}$, was changed by $0.5 \mathrm{~cm}$.

The method was also validated by intentionally miscalibrating the electromagnetic tracking system, point $\mathbf{C}$ in Fig. 5, by a known distance of $0.5 \mathrm{~cm}$ in the superior 


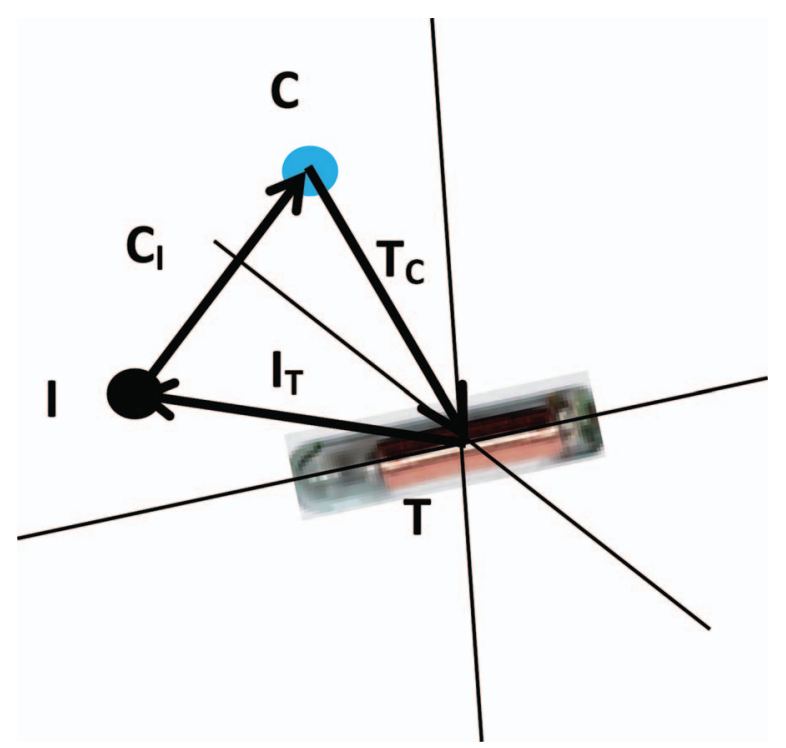

FIG. 5. Illustration of the vectors measured to find the offset between radiation isocenter and the origin of the RF tracking system. Three points of interest are shown: the origin of the RF tracking system, $\mathbf{C}$, radiation isocenter, $\mathbf{I}$ and the radiographic center of the coil in the transponder, $\mathbf{T}$. The position of C relative to $\mathbf{I}, \mathbf{C}_{\mathbf{I}}$, is found by adding the position of $\mathbf{T}$ relative to $\mathbf{C}, \mathbf{T}_{\mathbf{C}}$, is to the position of $\mathbf{I}$ relative to $\mathbf{T}, \mathbf{I}_{\mathbf{T}}$.

direction by displacing all calibration jigs and phantoms. The offset measured was, $\mathbf{C}_{\mathbf{I}}=(\mathrm{LR}, \mathrm{AP}, \mathrm{SI})=(0.002,0.048$, $0.431) \mathrm{cm}$, which was within the experimental uncertainty of this validation technique.

\section{C. Equipment and measurements}

Measurements were conducted on tracking systems installed in three separate treatment rooms. Two were on Varian
21EX treatment machines equipped with aS500 amorphous silicon portal imagers $(0.0784 \mathrm{~cm}$ pixel pitch), and will be referred to as EX1 and EX2. The third was on a Varian 21EX treatment machine with an aS1000 portal imager $(0.0392 \mathrm{~cm}$ pixel pitch), operated in a separate but affiliated clinic, which will be referred to as EX3. All three Calypso Systems were standard commercial configurations running version 2.0a application software. While the measurements described below were made using a Varian treatment machine, the methodology may be used on any treatment machine with a collimation system and a portal imager.

\section{RESULTS}

\section{A. Repeat measurements with the identical transponder position to determine uncertainty due to image noise and mechanical stability}

Figure 6 illustrates the variability in the measured collimator rotation axis rays from the image data and the variation in the offset between radiation isocenter and RF isocenter on consecutive measurements. The top and bottom rows show repeat measurements made on EX1 and EX2, respectively. Variations in determining the collimator rotation axes contribute to the variation in the measured size of the radiation isocenter uncertainty volume and therefore its center. Table I shows the size of radiation isocenter in each direction and its standard deviation for each of the measurements, as well as the vector components of the calibration offset for EX1. Table II shows the corresponding results for EX2. The standard deviation of the size of isocenter ranges from 0.004 to $0.012 \mathrm{~cm}$ in any given direction. The standard deviation in the components of the calibration offset range from 0.001 to $0.015 \mathrm{~cm}$.

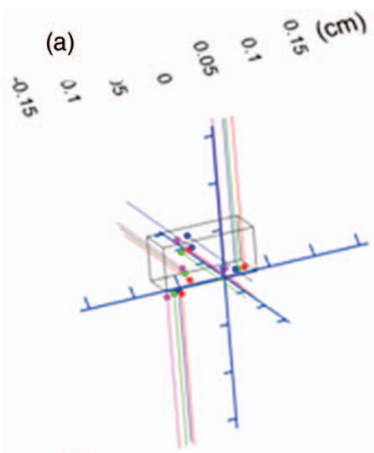

(d)

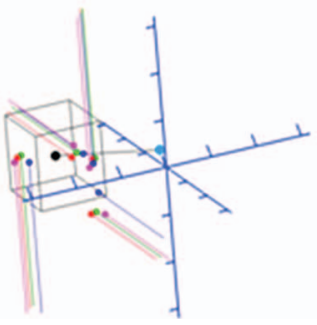

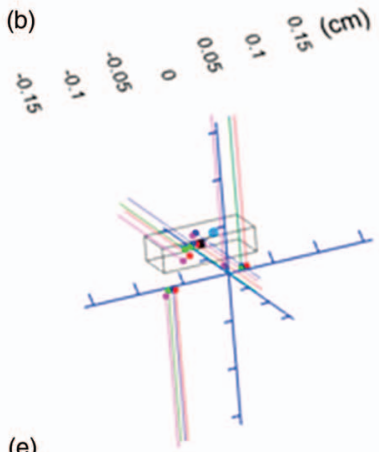

(e)

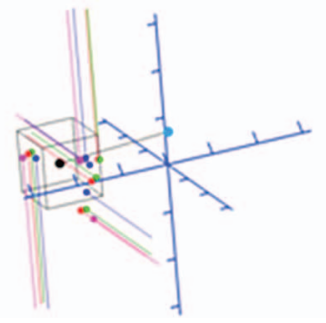

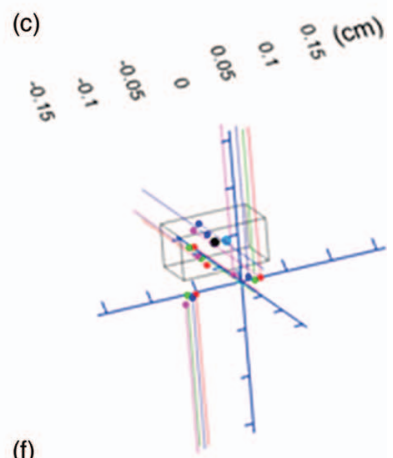

(f)

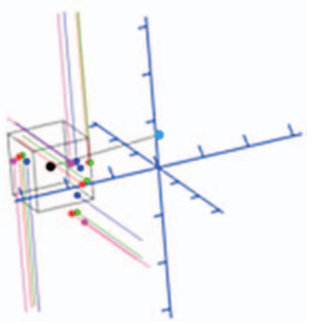

FIG. 6. Illustrations of repeat measurements. Figures (a)-(c) were obtained consecutively on one machine (EX1) and (d)-(f) on another machine (EX2) at the same institution. 
TABLE I. Results of three repeat measurements of the size of radiation isocenter and the offset to RF isocenter on EX1 (the transponder was not moved between successive measurements).

\begin{tabular}{lcccccccr}
\hline \hline \multirow{2}{*}{ Meas \# } & \multicolumn{3}{c}{ Isocenter size $(\mathrm{cm})$} & & \multicolumn{4}{c}{ Radiation to RF isocenter offset $(\mathrm{cm})$} \\
\cline { 2 - 7 } \cline { 6 - 9 } & LR & AP & SI & & LR & AP & SI & Distance \\
\hline 1 & 0.040 & 0.047 & 0.100 & & -0.010 & 0.007 & 0.011 & 0.017 \\
2 & 0.044 & 0.026 & 0.103 & & -0.014 & 0.014 & -0.009 & 0.022 \\
3 & 0.048 & 0.045 & 0.094 & & -0.011 & 0.003 & -0.009 & 0.014 \\
Ave & 0.044 & 0.039 & 0.099 & & -0.011 & 0.008 & -0.003 & 0.017 \\
$\sigma$ & 0.004 & 0.012 & 0.004 & & 0.002 & 0.006 & 0.011 & 0.004 \\
\hline \hline
\end{tabular}

\section{B. RF calibration offset results}

Figure 7 shows the size of the isocenter uncertainty volume in each direction (top) and the vector components and magnitude of the calibration offset (bottom) between radiation and RF isocenter after calibration of the tracking systems. Table III shows the average and standard deviation of these values. While there are variations from machine to machine, the magnitude of the calibration offset is always less than the manufacturer's stated accuracy of $0.2 \mathrm{~cm}$ using their standard calibration procedure, and ranged from 0.014 to $0.175 \mathrm{~cm}$. Both of these values occurred on EX1, which had the largest offset standard deviation $(0.036 \mathrm{~cm}$, primarily due to one outlier value) but smallest average offset $(0.053 \mathrm{~cm})$. Also, on EX1 the average vector components of the calibration offset were all within $0.021 \mathrm{~cm}$ of isocenter and smaller than the corresponding standard deviation. However, the EX2 showed systematic offsets in the LR component of the offset of $-0.068 \pm 0.019 \mathrm{~cm}$ and in the SI component of -0.092 $\pm 0.027 \mathrm{~cm}$, while EX3 had a notable systematic offset in the SI direction of $-0.072 \pm 0.008 \mathrm{~cm}$. The systematic offsets appearing on EX2 but not EX1 will be addressed more in Sec. 4.

It is interesting to examine the variation in the size of the radiation isocenter uncertainty volume because it contributes to the variation in the calibration offset. Nominally, the size of isocenter should be very stable. Typically, the standard deviation in the size of isocenter ranges between 0.006 and $0.013 \mathrm{~cm}$ with a notable outlier on EX1 in the SI direction of $0.025 \mathrm{~cm}$, as shown in Fig. 7 and Table III. Note from Table I that this is over six times larger than the variation found from three consecutive measurements performed on a single night $(0.004 \mathrm{~cm})$. It is also the only measurement of isocenter to show this large of a variation in any direction. The short-term variation of the technique reported in Tables I and II, and long-term variation results over 19 months reported in Table III and Fig. 7, indicate that the large variation in the SI direction on EX1 is almost certainly due to machine variation and not due to variation inherent to the measurement technique. Investigation of this outlier will be further addressed in Sec. 4.

\section{DISCUSSION}

The technique presented in this work was developed as a quality assurance tool for a multi-institutional protocol in which electromagnetic guidance was used for hypofractionated prostate treatments. One of the primary considerations in developing the technique was that it be easily portable to other institutions involved in our prostate SBRT protocol. The primary requirement that MV portal imaging be available on the treatment unit where electromagnetic tracking is used may be taken as a given in any modern treatment facility. Only a spare set of transponders and the ability to obtain individual transponder position data (possible through a research agreement with the manufacturer) are then needed for the measurements.

While the technique provides quantitative feedback on the accuracy with which the electromagnetic tracking system is calibrated to radiation isocenter it does not yet provide an easy way to improve the results aside from preforming a second calibration. One possibility would be to modify the tracking system to allow corrective offsets to be input after each monthly calibration and applied to the reported tracking results. However, input of the incorrect values could

TABLE II. Results of three repeat measurements of the size of radiation isocenter and the offset to RF isocenter on EX2 (the transponder was not moved between successive measurements).

\begin{tabular}{lcccccccr}
\hline \hline & \multicolumn{3}{c}{ Isocenter size $(\mathrm{cm})$} & & \multicolumn{4}{c}{ Radiation to RF isocenter offset $(\mathrm{cm})$} \\
\cline { 2 - 4 } \cline { 6 - 8 } Meas \# & LR & AP & SI & & LR & AP & SI & Distance \\
\hline 1 & 0.074 & 0.080 & 0.072 & & -0.085 & 0.014 & -0.079 & 0.117 \\
2 & 0.061 & 0.065 & 0.062 & & -0.087 & 0.041 & -0.085 & 0.128 \\
3 & 0.061 & 0.073 & 0.071 & & -0.085 & 0.038 & -0.080 & 0.123 \\
Ave & 0.066 & 0.072 & 0.068 & & -0.086 & 0.031 & -0.081 & 0.123 \\
$\sigma$ & 0.008 & 0.007 & 0.006 & & 0.001 & 0.015 & 0.003 & 0.006 \\
\hline \hline
\end{tabular}



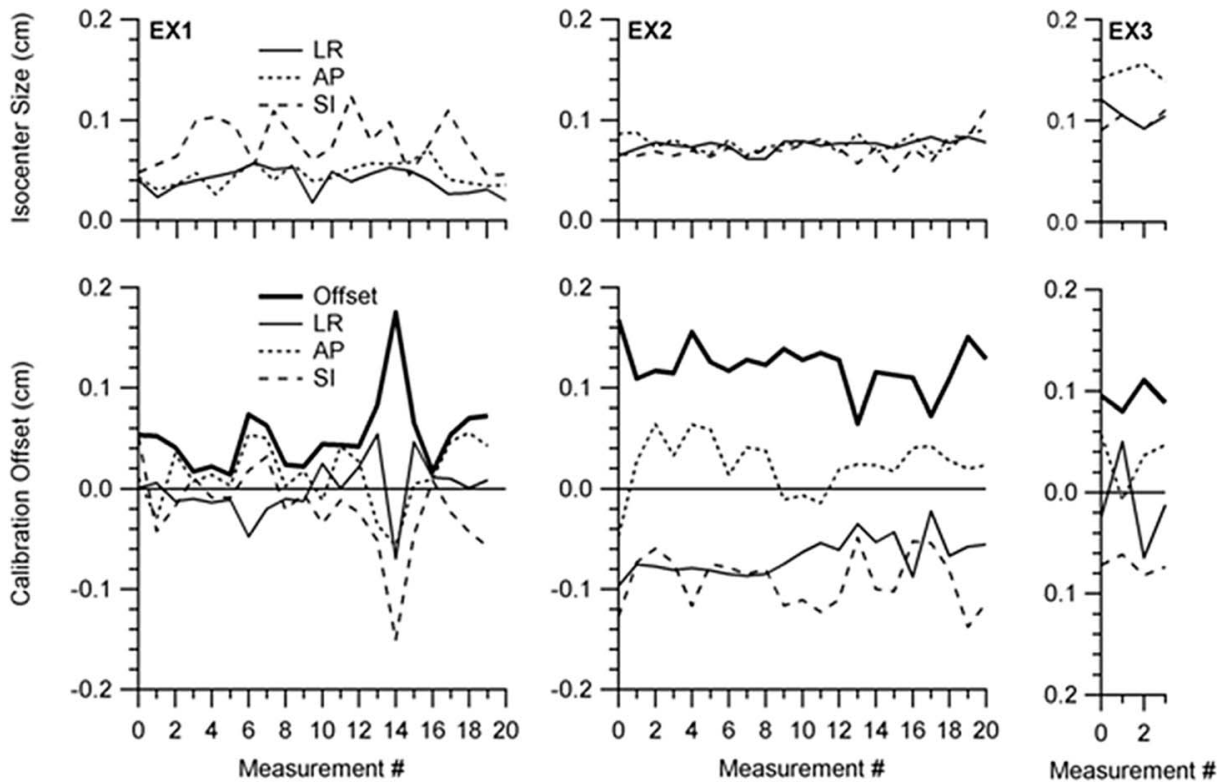

FIG. 7. The top row of graphs shows the size of radiation isocenter in each direction for each of the three machines. The bottom row of graphs shows the vector components and magnitude of the offset between radiation isocenter and the RF isocenter of each of the radiofrequency tracking systems.

itself become a possible source of error. Additionally, such an approach would only make sense if the calibration remains stable over the course of a month until the next system calibration.

It should be noted that the results reported here were primarily, but not exclusively, obtained immediately after the electromagnetic tracking systems were calibrated. Several of the measurements were made days or weeks after calibration. An attempt was made to look for drifts in the calibration and no significant correlation with changes over time were found. More data would be needed to draw any conclusions about the stability of the system's calibration with time.

In Sec. 3.B, it was noted that the calibration on EX2 and EX3 exhibited small systematic offsets (i.e., the average offset was roughly three standard deviations from zero) in one or more directions, while the EX1 did not exhibit such systematic offsets. In an attempt to investigate this observation, the calibration equipment from the tracking systems on EX1 and EX2 was swapped and the systems recalibrated several times. This did not change the calibration characteristics of either system indicating that the offset was not due to small differences in the calibration equipment, but was specific to the EX2 system, though the cause is not yet known.

One possible source of nonzero systematic vector offsets in the calibration may arise from the lasers being noncoplanar or nonorthogonal. Even if the lasers meet perfectly at isocenter, noncoplanar beams which are not perfectly orthogonal to isocenter will cause the calibration phantoms to be systematically offset when the phantoms are aligned to the lasers by their scribe lines which are many centimeters away from isocenter. However, the lasers on the EX2 system were found to be coplanar to better than $0.05^{\circ}$.

In Sec. 3.B, it was also noted that the size of isocenter in the SI direction on EX1 exhibited an unusually large variation compared to the other results. There were initial concerns that this was due to sensitivities in the image analysis to noise characteristics of the portal imager on that machine. However, because the noise in that imager was isotropic, there is no reason to believe the variation would be present in one direction and not the other. Additionally, internal consistency checks within the analysis software (such as the variation in finding

TABLE III. This table summarizes the average and standard deviation of the radiation isocenter size in each direction, and the vector components and magnitude of the offset between radiation isocenter and the calibrated origin of each radiofrequency tracking system.

\begin{tabular}{|c|c|c|c|c|c|c|c|c|}
\hline \multirow[b]{2}{*}{ Machine } & \multirow[b]{2}{*}{ Value } & \multicolumn{3}{|c|}{ Isocenter size $(\mathrm{cm})$} & \multicolumn{4}{|c|}{ Radiation to RF isocenter offset $(\mathrm{cm})$} \\
\hline & & LR & $\mathrm{AP}$ & SI & LR & AP & SI & Distance \\
\hline \multirow[t]{2}{*}{ EX1 } & Ave & 0.039 & 0.045 & 0.077 & -0.001 & 0.014 & -0.021 & 0.053 \\
\hline & $\sigma$ & 0.012 & 0.011 & 0.025 & 0.028 & 0.031 & 0.045 & 0.036 \\
\hline \multirow[t]{2}{*}{ EX2 } & Ave & 0.075 & 0.077 & 0.071 & -0.068 & 0.024 & -0.092 & 0.121 \\
\hline & $\sigma$ & 0.006 & 0.008 & 0.013 & 0.019 & 0.029 & 0.027 & 0.023 \\
\hline \multirow[t]{2}{*}{ EX3 } & Ave & 0.106 & 0.147 & 0.100 & -0.013 & 0.033 & -0.072 & 0.093 \\
\hline & $\sigma$ & 0.012 & 0.008 & 0.010 & 0.048 & 0.029 & 0.008 & 0.013 \\
\hline
\end{tabular}


the center of the transponder from one image to the next at a given gantry angle) did not indicate any image processing anomalies. It was further postulated that the variation was real and due to 12 years of wear in the rotation bearings of the collimator. A second independent analougous technique using a $1 \mathrm{~cm}$ stereotactic radiosurgery cone, a BRW target ball, and film was developed and implemented to test if the variation would also be observed. In one of five such measurements an anomaly in the position of the X1 field edge was noted at a collimator rotation of $315^{\circ}$ which caused a similar variation in the size of isocenter in the SI direction. However, the frequency of the variation did not match that seen in the technique presented here, so the results were not conclusive in identifying a mechanical issue as the source of the variation.

While it is not the primary intent of this technique to measure the size of the radiation isocenter uncertainty volume, it is a necessary intermediate step. It has been noted that the technique chosen here is more susceptible to graviational effects on collimation position than using a conventional star-shot technique with film. This is because the line drawn through isocenter from each spoke of the star-shot technique is taken as the center of the spoke, or the average postion of the two collimation edges. In contrast, our technique finds the largest rectilinear volume bounded by the rotation axis formed by each individual collimation edge. If the four collimator rotation rays found at each gantry angle were averaged together, it can be seen in Fig. 4, that the size of isocenter would always be smaller than shown in Fig. 7 and Table III. While this would provide a smaller and more consistent value in the size of radiation isocenter, it would also mask potential mechanical accuracy problems in the collimation system.

\section{CONCLUSIONS}

We have developed and validated a technique for independently measuring the offset between radiation isocenter and the calibrated origin of an electromagnetic tracking system. Results of the multi-institutional QA study will be presented separately when the study is complete. Validation measurements on three different tracking systems showed 3D offsets of $0.053 \pm 0.036,0.121 \pm 0.023$, and $0.093 \pm 0.013 \mathrm{~cm}$. All offsets were less than the $0.2 \mathrm{~cm}$ uncertainty stated by the manufacturer and specified by AAPM Task Group 147 on nonradiographic localization and positioning systems.

\section{ACKNOWLEDGMENT}

The authors have no conflict of interest.

\footnotetext{
a) Author to whom correspondence should be addressed. Electronic mail: litzen@umich.edu; Telephone: (734) 936-7831; Fax: (734) 936-7859.

${ }^{1}$ H. Lukka, C. Hayter, J. A. Julian, P. Warde, W. J. Morris, M. Gospodarowicz, M. Levine, J. Sathya, R. Choo, H. Prichard, M. Brundage, and W. Kwan, "Randomized trial comparing two fractionation schedules for patients with localized prostate cancer," J. Clin. Oncol. 23, 6132-6138 (2005).
}

${ }^{2}$ RTOG 9406, A phase I/II dose escalation study using three dimensional conformal radiation therapy for adenocarcinoma of the prostate (available URL: http://www.rtog.org/ClinicalTrials/ProtocolTable/ StudyDetails.aspx?study=9406).

${ }^{3}$ RTOG 0415, A phase III randomized study of hypofractionated 3DCRT/IMRT versus conventionally fractionated 3DCRT/IMRT in patients treated for favorable-risk prostate cancer (available URL: http://www.rtog.org/ClinicalTrials/ProtocolTable/StudyDetails.aspx? study $=0415$ ).

${ }^{4}$ H. M. Sandler, P. Y. Liu, R. L. Dunn, D. C. Khan, S. E. Tropper, M. G. Sanda, and C. A. Mantz, "Reduction in patient-reported acute morbidity in prostate cancer patients treated with 81-Gy intensity-modulated radiotherapy using reduced planning target volume margins and electromagnetic tracking: Assessing the impact of margin reduction study," Urology 75, 1004-1008 (2010).

${ }^{5}$ RTOG 0938, A randomized phase II trial of hypofractionated radiotherapy for favorable risk prostate cancer-RTOG CCOP study (available URL: http://www.rtog.org/ClinicalTrials/ProtocolTable/StudyDetails.aspx? study=0938).

${ }^{6}$ J. M. Balter, J. N. Wright, L. J. Newell, B. Friemel, S. Dimmer, Y. Cheng, J. Wong, E. Vertatschitsch, and T. P. Mate, "Accuracy of a wireless localization system for radiotherapy," Int. J. Radiat. Oncol., Biol., Phys. 61, 933-937 (2005)

${ }^{7}$ L. Santanam, K. Malinowski, J. Hubenshmidt, S. Dimmer, M. L. Mayse, J. Bradley, A. Chaudhari, K. Lechleiter, S. K. M. Goddu, J. Esthappan, S. Mutic, D. A. Low, and P. Parikh, "Fiducial-based translational localization accuracy of electromagnetic tracking system and on-board kilovoltage imaging system," Int. J. Radiat. Oncol., Biol., Phys. 70, 892-899 (2008).

${ }^{8}$ L. Santanam, C. Noel, T. R. Willoughby, J. Esthappan, S. Mutic, E. E. Klein, D. A. Low, P. J. Parikh, J. J. Gordon, A. J. Crimaldi, M. Hagan, J. Moore, and J. V. Siebers, "Quality assurance for clinical implementation of an electromagnetic tracking system," Med. Phys. 36, 3477-3486 (2009).

${ }^{9}$ This accuracy value is found in version 2.0 of the Calypso User Manual, section 1.7 (LBL0008-007)

${ }^{10} \mathrm{~K}$. R. Winston and W. Lutz, "Linear accelerator as a neurosurgical tool for stereotactic radiosurgery," Neurosurgery 22, 454-464 (1988).

${ }^{11}$ W. Lutz, K. R. Winston, and N. Maleki, "A system for stereotactic radiosurgery with a linear accelerator," Int. J. Radiat. Oncol., Biol., Phys. 14, 373-381 (1988).

${ }^{12}$ R. Nath, P. J. Biggs, F. J. Bova, C. C. Ling, J. A. Purdy, J. van de Geijn, and M. S. Weinhous, "AAPM code of practice for radiotherapy accelerators: Report of AAPM Radiation Therapy Task Group No. 45," Med. Phys. 21, 1093-1121 (1994)

${ }^{13}$ A. Gonzalez, I. Castro, and J. A. Martinez, "A procedure to determine the radiation isocenter size in a linear accelerator," Med. Phys. 31, 1489-1493 (2004).

${ }^{14}$ M. K. Woo, P. O'Brien, B. Gillies, and R. Etheridge, "Mechanical and radiation isocenter coincidence: An experience in linear accelerator alignment," Med. Phys. 19, 357-359 (1992).

${ }^{15}$ P. Skworcow, J. A. Mills, O. C. Haas, and K. J. Burnham, "A new approach to quantify the mechanical and radiation isocentres of radiotherapy treatment machine gantries," Phys. Med. Biol. 52, 7109-7124 (2007).

${ }^{16}$ P. Rowshanfarzad, M. Sabet, D. J. O'Connor, and P. B. Greer, "Isocenter verification for linac-based stereotactic radiation therapy: Review of principles and techniques," J. Appl. Clin. Med. Phys. 12, 185-195 (2011).

${ }^{17}$ P. Rowshanfarzad, M. Sabet, D. J. O'Connor, and P. B. Greer, "Investigation of the sag in linac secondary collimator and MLC carriage during arc deliveries," Phys. Med. Biol. 57, N209-N224 (2012).

${ }^{18}$ F. Rosca, F. Lorenz, F. L. Hacker, L. M. Chin, N. Ramakrishna, and P. Zygmanski, "An MLC-based linac QA procedure for the characterization of radiation isocenter and room lasers' position," Med. Phys. 33, 1780-1787 (2006).

${ }^{19} \mathrm{~W}$. Du and S. Gao, "Measuring the wobble of radiation field centers during gantry rotation and collimator movement on a linear accelerator," Med. Phys. 38, 4575-4578 (2011).

${ }^{20}$ T. Willoughby, J. Lehmann, J. A. Bencomo, S. K. Jani, L. Santanam, A. Sethi, T. D. Solberg, W. A. Tome, and T. J. Waldron, "Quality assurance for nonradiographic radiotherapy localization and positioning systems: Report of Task Group 147," Med. Phys. 39, 1728-1747 (2012).

${ }^{21}$ Varian Medical Systems, Palo Alto, CA (private communication). 